\title{
Variaciones estacionales en la morbimortalidad por tromboembolismo pulmonar en Galicia
}

\author{
J. MONTES SANTIAGO, G. REY GARCÍA', A. MEDIERO DOMÍNGUEZ ${ }^{1}$ \\ Servicio de Medicina Interna. ${ }^{1}$ Servicio de Documentación Clínica. \\ Hospital Meixoeiro. Vigo, Pontevedra
}

SEASONAL VARIATIONS IN MORBIDITY AND MORTALITY FOR PULMONARY EMBOLISM IN GALICIA, SPAIN

\section{RESUMEN}

Fundamento y objetivos: Analizar las variaciones estacionales en las hospitalizaciones y mortalidad por tromboembolismo pulmonar (TEP) en los hospitales del Servicio Gallego de Salud (SERGAS) durante el periodo 1996-2001.

Métodos: Se examinó el Grupo Relacionado de Diagnóstico (GRD) 78 (embolismo pulmonar) a partir del Conjunto Mínimo Básico de Datos (CMBD) de los Hospitales del SERGAS durante el sexenio mencionado, anotándose las características de edad, sexo, mes de alta y agrupación de estas en periodos estacionales.

Resultados: Se registraron 2.831 altas (44\% varones, estancia media global: 12,8 días, $70 \%>65$ años). La mediana mensual fue de 38 altas. La variación de los ingresos con respecto a esta fue de $+26 \%$ (diciembre) y $-25 \%$ (julio). Fueron significativamente más frecuentes las hospitalizaciones en otoño (OR:1,35, IC95\%:1,19-1,53) e invierno (OR:1,29, IC:1,14-1,46). La mortalidad fue mayor en primavera (OR:2, 18, IC:1,18$4,05)$, produciéndose el $83 \%$ de las muertes en pacientes $>65$ años.

Conclusiones: Se observó un marcado aumento de las hospitalizaciones por TEP durante los meses fríos y de la mortalidad en primavera. Dichas observaciones pueden tener trascendencia en la adopción de medidas preventivas eficaces.

PALABRAS CLAVE: Tromboembolismo pulmonar. Variación estacional.
ABSTRACT

Objectives: The goal of this report was to analyze the seasonal variation in the pulmonary embolism (EP) hospitalizations and mortality at the hospitals of the Galician Service of Health (SERGAS) in the Norwest of Spain, during the period 1996-2001.

Methods: The Related Group of Diagnosis (DRG) 78 (pulmonary embolism) from the SERGAS hospital discharge dataset during the 6year period was examined. Age, sex, month of discharge and seasonal period was annotated.

Results: A total of 2831 hospitalizations for TEP were recorded (44 $\%$ males, mean inhospital stay: 12,8 days, $70 \%>65$ years). Montly median was 38 discharges and the variations above and below this were $+26 \%$ (December) and -25\% (July), respectively. There were more frequents hospitalizations in autumn (OR:1.35, 95\% CI:1.19-1.53) and winter (OR: 1.29, CI:1.14-1.46). Mortality peaked in spring (OR:2.18, CI:1.18-4.05). Nearly $83 \%$ of these deaths occurred in persons $>75$ years old.

Conclusions: We observed a marked increase in EP hospitalizations during colder months and in mortality during spring. These observations may have implications in the adoption of preventive measures.

KEY WORDS: Pulmonary embolism. Seasonal variation.

Montes Santiago J, Rey García G, Mediero Domínguez A. Variaciones estacionales en la morbimortalidad por tromboembolismo pulmonar en Galicia. An Med Interna (Madrid) 2003; 20: 457-460.

\section{INTRODUCCIÓN}

El tromboembolismo pulmonar (TEP) es la tercera causa de muerte hospitalaria tras el ictus y la cardiopatía isquémica $(1,2)$. Para las dos últimas entidades se han descrito acusadas variaciones estacionales en los ingresos y muertes, con claro aumento en los periodos invernales (3-7). En el TEP y Trombosis venosa profunda (TVP) los datos son más escasos y se han publicado observaciones contradictorias en otros países con respecto a su aumento en los meses fríos o a la mayor mortalidad durante estos (8-11).

En nuestro país son escasos los estudios sobre dicho tema, habiéndose comunicado recientemente la falta de estacionali- dad de TVP y TEP en una comunicación a partir de un único centro en un periodo de 4 años (marzo 1998-febrero 2002) (12). En este estudio analizamos las admisiones hospitalarias y la mortalidad por TEP en el Servicio Gallego de Salud (SERGAS) durante un periodo de 6 años (1996-2001).

\section{MATERIAL Y MÉTODOS}

A partir del Conjunto Mínimo Básico de Datos de Hospitalización de Agudos (CMBD-HA) de los hospitales del SERGAS y Policlínico Vigo S.A. (hospital concertado que atiende

Trabajo aceptado: 28 de abril de 2003

Correspondencia: Julio Montes Santiago. Servicio de Medicina Interna. Hospital Meixoeiro. C/ Meixoeiro, s/n. 36200. Vigo. e-mail: julio.montes.santiago@ sergas.es 
a un sector público de población) se estudió la evolución del Grupo Relacionado de diagnóstico (GRD) 78 (Embolismo pulmonar) en el periodo 1996-2001 (13), agrupándose las altas por edad, meses y variación estacional (invierno: eneromarzo, primavera: abril-junio, verano: julio-septiembre, otoño: octubre-diciembre). Es preciso notar que los Servicios hospitalarios del SERGAS mantienen una tasa de altas válidas codificadas $>96 \%$ desde el año 1996. La comparación de los resultados obtenidos en los distintos periodos estacionales se realizó por el método chi-cuadrado y se calcularon los riesgos relativos (Odds ratios, OR) e intervalos de confianza (IC) del 95\% por el método de Mantel-Haenszel.

\section{RESULTADOS}

Se registraron un total de 2.831 altas (646 en primavera, 630 en verano, 791 en otoño y 764 en invierno). El 44\% (1.253) fueron varones y se contabilizó una estancia media global de 12,8 días [rango: 11,3 (septiembre)-14,5 (mayo)]. El $91 \%$ de los casos fue $>45$ años y el $70 \%>65$ años (IC: 68$71 \%$ ). La mortalidad global fue del $4.9 \%$ [rango: 2,2 (marzo)8,2 (abril)], produciéndose el $83 \%$ en $>65$ años (IC: $77-89 \%$ ). En la figura 1 se representa la curva mensual (mediana de los meses del periodo) y en la figura 2 la curva estacional de hospitalizaciones y mortalidad. La mediana mensual de altas fue de 38 casos [rango: 29 (julio)-48 (diciembre)]. La variación entre la mediana de estos meses con menor y mayor número de altas fue, pues, del $67 \%$ y la variación con respecto a la mediana mensual de $-25 \%$ (julio) y $+26 \%$ (diciembre), respectivamente. Fueron significativamente más frecuentes las hospitalizaciones durante los periodos estacionales fríos respecto al verano [otoño: OR:1,35 (IC:1,19-1,53); invierno: OR:1,29 (IC:1,14-1,46)]. La mortalidad en primavera y otoño fue mayor que la del verano [primavera: OR: 2,18 (IC:1,184,05); otoño: OR: 1,90 (IC:1,02-3,55)].

\section{DISCUSIÓN}

La publicación de las estadísticas del CMBD hospitalarios de las diversas comunidades autónomas y del Sistema Nacional de Salud (SNS), así como su fácil accesibilidad a través de Internet, están permitiendo obtener valiosos datos epidemiológicos sobre ciertos procesos patológicos. En muchos de estos sólo disponíamos de estimaciones indirectas mediante extrapolación de los datos de otros países o las informaciones eran muy incompletas. Por ejemplo, en series clínicas recientes sobre TEP en España se soslaya sistemáticamente el tema de la variación estacional (14-16). Por último, las referencias proporcionadas por algunas publicaciones muy fragmentarias, locales o con escasos pacientes bien pudieran inducir a conclusiones erróneas. En este sentido hemos encontrado en Galicia un claro predominio de las hospitalizaciones de los pacientes con TEP en otoño e invierno, lo cual contradice la comunicación reciente procedente de un solo hospital (Getafe, Madrid) (12) con limitado número de casos (115 TEP) y que no encontraba estacionalidad en los ingresos por TEP. Sin embargo, la gráfica mensual de altas para el GRD 78 -que ha sido publicada aunque sin detalles-, realizada a partir de los datos del SNS del año 1999 (4872 casos totales de TEP), permite apreciar un claro pico en diciembre ( $>485$ casos) y un valle en junio ( $<340$ casos) (17). Ello parece confirmar para el resto del estado español, y a semejanza de otros países como Francia (11), la tendencia encontrada en nuestro estudio.

La explicación de esta estacionalidad en los ingresos, y a semejanza de lo que sucede con otras entidades, por ejemplo el ictus o infarto de miocardio $(5,7)$ o la insuficiencia cardiaca (18-20), permanece oscura y no se ha estudiado específicamente. Se ha sugerido que la vasoconstricción mediada por el frío y la actividad física reducida podría disminuir el flujo sanguíneo en las extremidades inferiores, favoreciendo así la trombosis en estos (11). Se han descrito así mismo algunas variaciones esta-

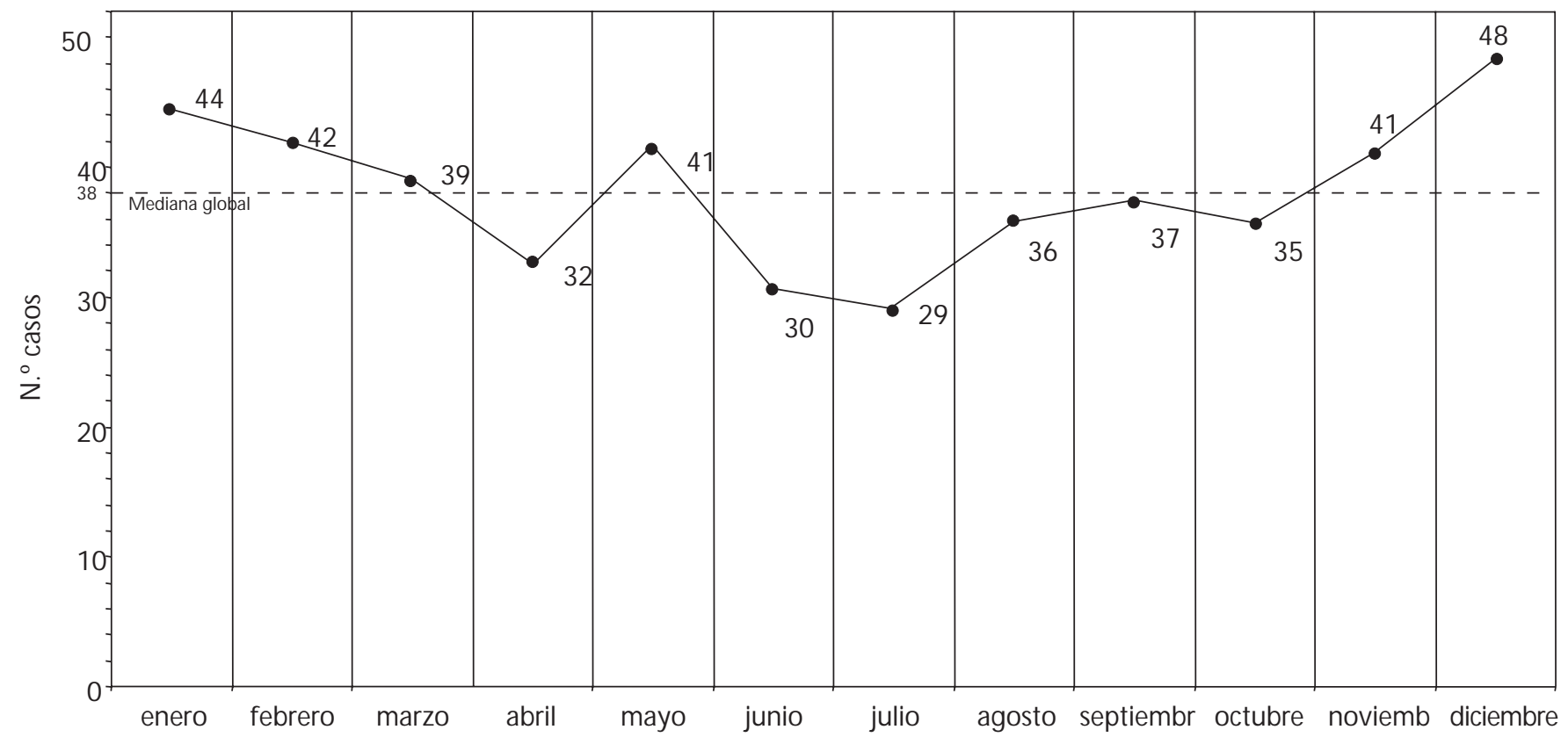

Fig. 1. M ediana mensual de casos de tromboembolismo pulmonar (GRD 78) en el periodo 1996-2001. 


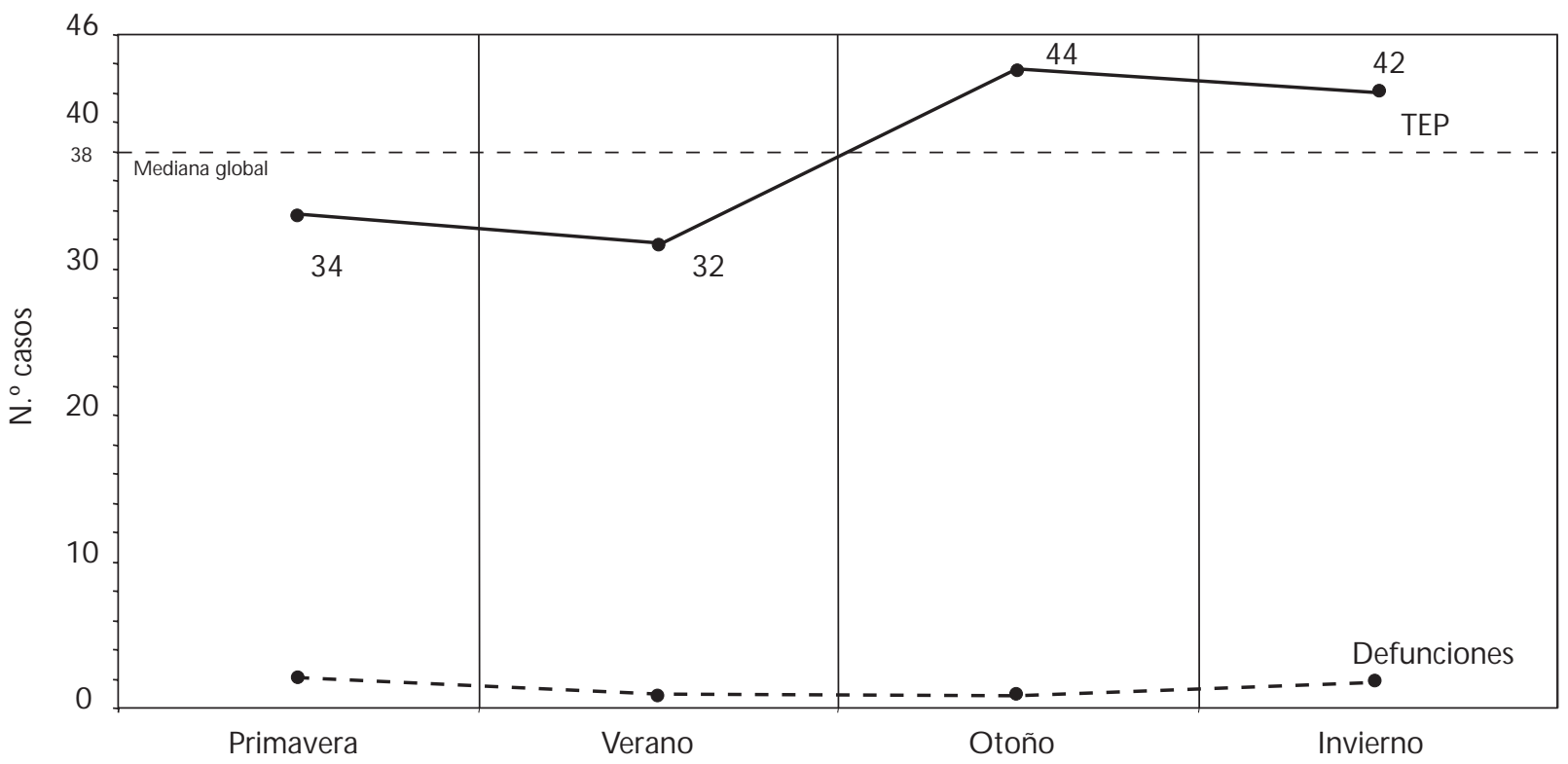

Fig. 2. Evolución de casos de TEP (GRD 78) y mortalidad en Galicia (mediana estacional del periodo 1996-2001).

cionales en ciertos factores de la coagulación que se han puesto en relación con infecciones invernales $(21,22)$.

Otro dato que hemos encontrado en nuestro estudio es la existencia de una mayor mortalidad en primavera, en consonancia con datos publicados procedentes de autopsias de muertes por TEP masivos (23). Tampoco se ha encontrado una explicación convincente para este dato aunque se ha sugerido que podría deberse a las comorbilidades asociadas con los climas fríos, razón también invocada en otras patologías cardiovasculares $(3,6,24)$.

La vacunación antigripal, aunque no específicamente estudiada en el TEP, podría constituir una buena medida indirecta de prevención de tal entidad $(25,26)$, teniendo en cuenta la preferente afectación de los ancianos (27-29), su incidencia notablemente aumentada en pacientes hospitalizados respecto a los ambulatorios (28) y dada su efectividad en la reducción de muerte y hospitalización por otros procesos cardiovasculares con fuerte componente estacional, como el infarto de miocardio (31) y la insuficiencia cardiaca (32).

En resumen, hemos encontrado en Galicia un aumento de las hospitalizaciones otoñales e invernales por TEP, habiéndose encontrado para dicha entidad una mayor mortalidad primaveral. Dichas observaciones pudieran tener importantes consecuencias para la prevención.

\section{AGRADECIMIENTOS}

Agradecemos a los doctores Carmen Sixto y Víctor del Campo las sugerencias realizadas en la elaboración de este trabajo.

\section{Bibliografía}

1. Ruiz Manzano J, Alberich Sotomayor P, Blanquer Olivas JM, Capelastegui Sáiz A, Cabezudo Hernández MA, De Gregorio Tariza MA et al. Profilaxis de la enfermedad tromboembólica venosa. Recomendaciones SEPAR, 1996. (Disponible en http://www.separ.es/index/htm, acceso $10 / 12 / 2002)$

2. Stein PD, Huang HL, Afzal A, Noor HA. Incidence of acute pulmonary embolism in a General Hospital. Relation to age, sex and race. Chest 1999; 116: 909-13.

3. The Eurowinter Group. Cold exposure and winter mortality from ischaemic heart disease, cerebrovascular disease, respiratory disease, and all causes, in warm and cold regions of Europe. Lancet 1997; 349: 1341-6.

4. Spencer FA, Goldberg RJ, Becker RC, Gore JM. Seasonal distribution of acute myocardial infarction in the Second National Registry of Myocardial Infarction. J Am Coll Cardiol 1998; 106: 649-53.

5. Sheth T, Nair C, Muller J, Yusuf S. Increased winter mortality from acute myocardial infarction and stroke: the effect of age. J Am Coll Cardiol 1999; 33: 1916-9.

6. Lanska DJ, Hoffmann RG. Seasonal variation in stroke mortality rates. Neurology 1999; 52: 984-90.

7. Oberg AL, Ferguson JA, McIntyre LM, Horner RD. Incidence of stroke and season of the year: evidence of an association. Am J Epidemiol 2000; 152: 558-64.

8. Manfredini R, Gallerani M, Salmi R, ZamboniP, Fersini C. Fatal pulmonary embolism in hospitalized patients: evidence for a winter peak. J Int Med Res 1994; 2: 85-9.

9. Allan TM, Douglas AS. Seasonal variation in deep vein thrombosis. Fatal pulmonary embolism is increased in both autumn and winter. BMJ 1996; 312: 1227

10. Bilora F, Manfredini R, Petrobelli F, Vettore G, Boccioletti V, Pomerri F. Chronobiology of nonfatal pulmonary thromboembolism. Panminerva Med 2001; 43: 7-10.

11. Boulay F, Berthier F, Schoukroun G, Raybaut C, Gendreike Y, Blaive B Seasonal variation in hospital admission for deep vein thrombosis and pulmonary embolism: analysis of discharge data. BMJ 2002; 323: 601-2. 
12. Barba R, Flores Y, Losa JE, Guijarro C, Martín H, Espinosa A et al. Estacionalidad y enfermedad tromboembólica. Rev Clin Esp 2002; (Supl. 1): 363.

13. Conxunto Mínimo Básico de Datos. Hospitais do Servicio Galego de Saúde. SERGAS. Secretaría Xeral. Subdirección Xeral de Información Sanitaria. Xunta de Galicia.

14. Saro G, Campos JF, Hernández JM, Anta M, Olmos JM, GonzálezMacías et al. Diagnostic approach to patients with suspected pulmonary embolism: a report from the real world. Postgrad Med J 1999; 75: 285-9.

15. López-Beret P, Pinto JM, Romero A, Orgaz A, Fontcuberta J, Oblas M. Systematic study of occult pulmonary thromboembolism in patients with deep venous trhombosis. J Vasc Surg 2001; 33: 515-21.

16. Calvo Romero JM, Arrebola García JD, Magro Ledesma D, Ramos Salado JL, Bureo Dacal JC, Bureo Dacal P et al. El espectro clínico actual del tromboembolismo pulmonar. Rev Clin Esp 2002; 202: 130-5.

17. Sistema Nacional de Salud. Año 1999. Explotación de bases del CMBD. Estadísticos de referencia estatal de los sistemas de agrupación de registros de pacientes (G.D.R.). Ministerio de Sanidad y Consumo, Subdirección general de Análisis Económico y Estadística; Madrid 2002; GDR 78, p. 201.

18. Boulay F, Berthier F, Systeron O, Gendreike Y, Gibelin P. Seasonal variation in chronic heart failure hospitalizations and mortality in France. Circulation 1999; 100: 280-6.

19. Montes Santiago J, Rey García G, Mediero Domínguez A, González Vázquez L, Pérez Fernández E, Del Campo Pérez V et al. Variaciones estacionales en la hospitalización y mortalidad por insuficiencia cardiaca crónica en Vigo. An Med Interna (Madrid) 2001: 18: 578-81.

20. Stewart S, McIntyre K, Capewell S, McMurray JV. Heart failure in a cold climate: seasonal variation in heart failure-related morbidity and mortality. J Am Coll Cardiol 2002; 39: 760-6.

21. Woodhouse PR, Khaw KT, Plummer M, Foley A, Meade TW. Seasonal variations of plasma fibrinogen and factor VII activity in the elderly: winter infections and death from cardiovascular disease. Lancet 1994, 343: 435-9.
22. Luong TH, Rand JH, Wu XX, Golbold JH, Gascon-Lema M, Tuhrin S. Seasonal distribution of anthiphospolipid antibodies. Stroke 2001; 32: 1707-11.

23. Green J, Edwards C. Seasonal variation in the necropsy incidence of massive pulmonary embolism. J Clin Pathol 1994; 47: 58-60.

24. Donaldson GC, Keatinge WR. Excess winter mortality: influenza or cold stress? Observational study. BMJ 2002; 324: 89-90.

25. Nichol KL, Wuorenma J, von Stenberg T. Benefits of influenza vaccination for low, intermediate and high-risk senior citizens. Arch Intern Med 1998; 158: 769-76.

26. Couch RB. Prevention and treatment of influenza. N Engl J Med 2000; 343:1778-87.

27. Kniffin WD Jr, Baron JA, Barrett J, Birkmeyer JD, Anderson FA Jr. The epidemiology of diagnosed pulmonary embolism and deep venous thrombosis in the elderly. Arch Intern Med 1994; 154: 861-6.

28. Martín Graczyk A, Bugidos Benavides R. Tromboembolismo venoso en el anciano. Importancia, epidemiología y fisiopatología. En: Veiga Fernández F, coordinador. Tromboembolismo venoso en el anciano. Barcelona: Glosa ediciones; 2000; 9-17.

29. Monreal M. Epidemiología, patogenia e historia natural del tromboembolismo venoso. En: Rocha Hernando E, Martínez Brotons F, Monreal Bosch M, editores. Manejo práctico del paciente con tromboembolismo venoso. Madrid: Acción Médica, 2002; 1-10.

30. Heit J, Melton LJ, Lohse CM, Petterson TM, Silverstein MD, Mohr DN et al. Incidence of venous thromboembolism in hospitalized patients vs. community residents. Mayo Clin Proc 2001; 76: 1102-10.

31. Gurfinkel E, Mautner B. Prevención secundaria de la cardiopatía isquémica con la vacunación antigripal. Nueva evidencia sobre el papel de la infección y los síndromes coronarios agudos. Rev Esp Cardiol 2002; 55 : 1009-12.

32. Kaiser L, Hayden FG. Hospitalizing influenza in adults. Curr Clin Trop Infect Dis 1999; 19: 112-34. 